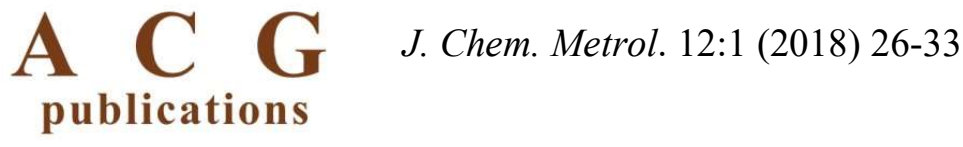

\title{
Determination of taxanes by validated LC-MS/MS method in hazelnut collected from different regions and altitudes in Turkey
}

\author{
Sibel Bayıl Oguzkan $\odot^{1}$, Bora Karagul $\odot^{2}$, Elif Sine Aksoy $\odot^{2}$, \\ Ayşe Uzun $\odot^{2}$, Merve Can $\odot^{2}$, Hasibe Yilmaz $\odot^{\circledR}$, \\ Halil Ibrahim Ugras $\odot^{2^{*}}$, Burcu Binici $\odot^{3^{*}}$ and Ahmet C Goren $\odot^{3}$ \\ ${ }^{1}$ Gaziantep University, Arts and Science Faculty, Biology Department, Gaziantep, Türkiye \\ ${ }^{2}$ Düzce University, Science and Arts Faculty, Chemistry Department Düzce, Türkiye \\ ${ }^{3}$ TUBITAK UME, Chemistry Group, Organic Chemistry Laboratory, Gebze, Kocaeli, \\ Türkiye
}

(Received April 23, 2018; Revised May 13, 2018; Accepted May 23,2018)

\begin{abstract}
In this study, mass fractions of paclitaxel, cephalomannine, 10-DAB III and baccatin III in hazelnut were measured with new and validated LC-MS/MS method. Green outer nut husk and nutshell of Turkish hazelnut (Corylus colurna L.) were collected from Düzce, Trabzon, Adapazarı and Samsun at several altitudes during August and September in 2014. The target analytes were prepared using solid-liquid extraction and analysed by LC-MS/MS. High amount of baccatin III, used for synthesizing semi-synthetic paclitaxel, was found in the nutshells collected at 250-500 m altitude in Cumayeri district of Duzce. In the husk samples from Vakfikebir district of Trabzon, the highest amount of baccatin III was obtained. The mass fractions of baccatin III in nutshell and husks were between 164.38-1020.85 $\mu \mathrm{gkg}-1$ and 166.12-923.64 $\mu \mathrm{gkg}-1$, respectively.
\end{abstract}

Keywords: Taxanes; baccatin III; hazelnut; LC-MS/MS; method validation. (C) 2018 ACG Publications. All rights reserved.

\section{Introduction}

Taxol, an anticancer drug having high importance, is used for treatment of ovarian, breast, lung and skin cancers. It can be administered directly or together with some chemotherapeutic agents [1]. Although total synthesis of taxol has now been realized by different groups [2-5], their approaches are expensive. Therefore, currently, various studies have been concentrated on determination of taxol in different sources. For instance, in one of the studies, a high proportion $(0.1 \%)$ of 10 -deacetylbaccatin III (10-DAB III) was obtained from renewable foliage of the yew (Taxus baccata L.) without damaging the tree. Also, in Turkey, paclitaxel (Taxol A) from Taxus baccata L. trees was used in treatment of ovarian and breast cancers. On the other hand, disappointingly, because of its complex structure, low amount of taxol exists in trees. Therefore, alternative sources for supplying the drug should be investigated [6].

Semi synthesis of taxol is the other way of obtaining taxol, which involves derivation of taxol via biotransformation of baccatin III and 10-DAB III in Taxus baccata leaves [7,8]. These compounds were then used in the preparation of medicaments [9].

\footnotetext{
*Corresponding authors E-Mail: halilugras@duzce.edu.tr; burcu.binici@tubitak.gov.tr
} 
Moreover, applying a solid-phase extraction method using 30\% methanol, 10-DAB III was detected as $0.0566 \%$ and $0.0695 \%$ in Taxus baccata leaves (needles) and twigs, respectively [10]. Ballero et al. collected samples from 11 different regions in Italy during the summer season to perform a study for determination of taxane and 10-DAB III in foliage of Taxus baccata L. Although paclitaxel was not detected, 10-DAB III and taxane were determined in the range of $243-1665 \mathrm{mg} / \mathrm{kg}$ and $0-300$ $\mathrm{mg} / \mathrm{kg}$, respectively [11,12].

This study presents new sources of paclitaxel and its derivatives from performing measurements on nutshells and husks. In order to analyze hazelnut samples, a reliable sample preparation and LCMS/MS method were developed and validated herein. All validation and uncertainty calculation steps of developed method were assessed.

\section{Material and Method}

\subsection{Chemicals}

Cephalomannine analytical standard (4), baccatin III (3) ( $\geq 95 \%$ ), 10-deacetylbaccatin III (2) ( $\geq$ $95 \%$ ), paclitaxel reference standard (1) and erythromycin neat materials (Figure 1) were obtained from Sigma Aldrich (USA). Methanol, ethanol, acetone and dichloromethane, all HPLC grade, $>99.9 \%$, were purchased from Merck (Darmstadt, Germany).

\subsection{Plant Material}

Green outer nut husk and nutshell samples of Turkish hazelnut (Corylus colurna L.) were collected from different regions (Trabzon, Adapazar1, Samsun and Düzce) (Figure 2) from several altitudes such as 0-250 m, 250-500 m, 500+ m (Table 1) during August and September 2014 and. The samples were dried under sun. The dried samples were milled and sieved by passing through 80 -mesh sieves. The finalized powder samples were filled into amber glass bottles and kept at $+4{ }^{\circ} \mathrm{C}$ to prevent decomposition of the analytes by light and heat untill measurement time .

\subsection{Preparation of calibration and working solutions}

Two stock solutions containing cephalomannine, baccatin III, 10-deacetylbaccatin III and paclitaxel were prepared at $1000 \mathrm{mg} / \mathrm{kg}$ and $1 \mathrm{mg} / \mathrm{kg}$ mass fraction levels in methanol. Internal standard stock solutions were also prepared at $1000 \mathrm{mg} / \mathrm{kg}$ and $1 \mathrm{mg} / \mathrm{kg}$ mass fraction levels in methanol. Erythromycin was used as an internal standard. While the native stock solution at $1000 \mathrm{mg} / \mathrm{kg}$ level was used to prepare $1 \mathrm{mg} / \mathrm{kg}$ stock solution, the $1 \mathrm{mg} / \mathrm{kg}$ stock solution was used to prepare calibration solutions at six mass fraction levels between 10 and $1000 \mu \mathrm{g} / \mathrm{kg}$. The mass fraction of internal standard was kept constant at each level $100 \mu \mathrm{g} / \mathrm{kg}$.

\subsection{Sample preparation}

$10 \mathrm{~g}$ grinded sample was placed into a volumetric flask and $100 \mathrm{mg}$ of $1 \mathrm{mg} / \mathrm{kg}$ internal stock solution was added. Acetone at $1: 10(\mathrm{~g} / \mathrm{mL})$ ratio was mixed with sample and solid-liquid extraction was performed by shaking the sample on a shaker for 72 hours for nutshell and 48 hours for nut husk. Then, the sample was filtered by double-layer filter paper into the amber glass vial and the solvent part was removed under gentle stream of nitrogen. $50 \mathrm{mg}$ of the extract was dissolved in $1 \mathrm{~g}$ of $\mathrm{MeOH}: \mathrm{H}_{2} \mathrm{O}$ $(70: 30, \mathrm{v} / \mathrm{v})$ in a volumetric flask and kept in an ultrasonic bath for $1 \mathrm{~h}$. Then, it was centrifuged at 4800 $g$ for 30 minutes. An aliquot of methanol extract was purified by filtering through a $0.2 \mu \mathrm{m}$ filter before being measured in LC-MS/MS.

\subsection{Chromatographic Conditions}

A HPLC (Zivak $\left.{ }^{\circledR}\right)$ and Zivak ${ }^{\circledR}$ Tandem Gold Triple quadrupole (Istanbul, Turkey) mass spectrometer, having a Phenomenex Synergi 4u Max-RP 80A column ( 150 x $2.0 \mathrm{~mm}$ i.d., $4 \mu \mathrm{m}$ particle size) was used to analyze the hazelnut samples. The mobile phase A was prepared as $0.1 \%$ formic acid in methanol-water $(70: 30, \mathrm{v} / \mathrm{v})$ mixture and an isocratic program was applied by using $100 \%$ of mobile phase A for 0-10.00 minutes long. Flow rate of the mobile phase was $0.30 \mathrm{~mL} / \mathrm{min}$ and the temperature of column was set to $30^{\circ} \mathrm{C}$. The volume of the injection was $20 \mu \mathrm{L}$. The chromatograms of the analytes 
Determination of taxanes by validated LC-MS/MS method

are given in Figure 3. The optimum ESI parameters were determined as 2.40 mTorr CID for gas pressure, $5000.00 \mathrm{~V}$ ESI for needle voltage, $600.00 \mathrm{~V}$ ESI for shield voltage, $300^{\circ} \mathrm{C}$ for drying gas temperature, $50{ }^{\circ} \mathrm{C}$ for API housing temperature, $55 \mathrm{psi}$ for Nebulizer gas pressure and $40 \mathrm{psi}$ for drying gas pressure. The parent, daughter ions and collision energy values are included in Table 2.

\subsection{Method Validation}

In validation process linearity, limit of detection (LOD), limit of quantification (LOQ) and repeatability (in a day and intra days) and recovery parameters were evaluated [13-15].

\subsubsection{Linearity}

The linearity of method was evaluated by preparing calibration solutions at six mass fraction levels between 10 and $1000 \mu \mathrm{gkg}^{-1}$ values and based on the matrix matched and internal standard calibration techniques. The mass fraction value of internal standard erythromycin was kept constant at each level at $100 \mu \mathrm{gkg}^{-1}$. Also the same amount of blank matrix extract was added to all calibration solutions to perform matrix matching calibration technique. Calibration graph was drawn as the ratio of peak area of native and internal standard compounds versus the ratio of mass fraction of native and internal standard compounds. The correlation coefficients, $R^{2}$, and linear equations were determined and presented in Table 3 .

\subsection{2. $L O D$ and $L O Q$}

In order to calculate LOD and LOQ values, the mass fraction of the sample was divided by signal to noise $(\mathrm{S} / \mathrm{N})$ ratio and then obtained value was multiplied by 3 and 10 to obtain LOD and LOQ, respectively [14-16]. The values are presented in Table 4 in $\mu \mathrm{gkg}^{-1}$ unit.

\subsubsection{Repeatability}

The repeatability was evaluated by applying the method in triplicate at three different concentration levels in a day and in three different days. The mean of relative standard deviations of all concentration levels were calculated. The value of relative standard deviation is presented in Table 4 .

\subsubsection{Recovery}

For recovery tests, the blank nut samples were spiked by standard solution at three concentration levels in the working range and method was applied in triplicate for each level. The recovery values were calculated by Equation 1. and is presented in Table 4.

$$
R \%=\frac{C_{o b s}-C_{\text {blank }}}{C_{S p k}} \times 100
$$

$R \% \quad$ : Percentage of recovery

$C_{o b s}:$ Concentration observed by method application

$C_{\text {blank }}$ : Concentration of analyte in the blank sample

$C_{s p k}$ : Theoretical concentration of spiked sample

\subsection{Evaluation of Uncertainty}

The uncertainty parameters were determined as weighing of sample intake, final sample and addition of internal stock solution, preparation of native stock solution, repeatability, recovery and calibration curve [16].

\subsubsection{Uncertainty of weighing of sample intake, final sample and addition of internal stock solution}

Combined standard measurement uncertainty of weighing the first and last samples and internal stock solution added to the sample were calculated separately using Equation 2, where $u_{\text {cal }}$ was obtained from the manufacturer's certificate of the balance. This value was used for both sample and tare.

$$
u(W)=\sqrt{\left(u_{\text {Calsample }}\right)^{2}+\left(u_{\text {Caltare }}\right)^{2}}
$$


where,

$u(W)$ : Combined standard measurement uncertainty of weighing

$u_{\text {Cal-sample: }}$ Standard measurement uncertainty, calibration of balance, sample

$u_{\text {Cal-tare: }}$ Standard measurement uncertainty, calibration of balance, tare

\subsubsection{Uncertainty of preparation of native stock solution}

Combined standard measurement uncertainty of native stock solution, which were prepared gravimetrically, was calculated using Equation 3, where $u(P)$ was obtained from manufacturer's certificate of the standard solution.

$$
\frac{u\left(C_{S S}\right)}{C_{S S}}=\sqrt{\left(\frac{u(P)}{P}\right)^{2}+\left(\frac{u(W)}{W}\right)^{2}}
$$

where,

$u\left(C_{S S}\right)$ : Combined standard measurement uncertainty of standard stock solution

$u(P)$ : Standard measurement uncertainty, purity, standard solution

$u(W)$ : Standard measurement uncertainty, weighing standard solution (Eq. 4)

$C_{S S}$ : Concentration of standard stock solution

$P$ : Purity of standard stock solution

$W$ : Weight of standard stock solution

$$
u(W)=\sqrt{\left(u_{\text {Calsample }}\right)^{2}+\left(u_{\text {Calsolvent }}\right)^{2}+\left(u_{\text {Caltare }}\right)^{2}}
$$

where,

$u(W)$ : Combined standard measurement uncertainty, weighing

$u_{\text {Cal-sample: }}$ Standard measurement uncertainty, calibration of balance, sample

$u_{\text {Cal-solvent }}$ : Standard measurement uncertainty, calibration of balance, solvent

$u_{\text {Cal-tare: }}$ Standard measurement uncertainty, calibration of balance, tare

\subsubsection{Uncertainty of calibration graph}

The combined standard measurement uncertainty from calibration graph was calculated using Equation 5 [17].

$$
u\left(c_{0}\right)=\frac{S}{B_{1}} \sqrt{\frac{1}{p}+\frac{1}{n}+\frac{\left(c_{0}-\bar{c}\right)^{2}}{S_{x x}}} \quad S x x=\sum_{i=1}^{n}\left(c_{i}-\bar{c}\right)^{2}
$$

where,

$u\left(c_{0}\right)$ : Combined standard measurement uncertainty of calibration curve

$S$ : Residual standard deviation

$B_{1}$ : Slope

$p$ : Number of measurement to determine $\mathrm{c}_{0}$

$n$ : Number of measurement for calibration

$c_{0}$ : Determined concentration of the sample by using calibration curve

$c$ : Average value of the different calibration solution concentrations 
Determination of taxanes by validated LC-MS/MS method

\subsubsection{Uncertainty of repeatability}

The standard measurement uncertainty from repeatability of the method was calculated by using Equation 6.

$$
u(r)=\frac{R S D}{\sqrt{n}}
$$

where,

$u(r)$ : Standard measurement uncertainty of repeatability

$R S D$ : Relative standard deviation

$n$ : Number of sample

\subsubsection{Uncertainty of recovery}

The standard measurement uncertainty from recovery of the method was calculated by using Equation 7.

where,

$$
\begin{gathered}
u\left(R_{m}\right)=R_{m} \sqrt{\left(\overline{\left.\frac{u\left(C_{o b s}\right)}{\overline{C_{o b s}}}\right)^{2}+\left(\frac{u\left(C_{S p k}\right)}{C_{S p k}}\right)^{2}}\right.} \\
R_{m}=\frac{\overline{C_{o b s}}}{C_{c e r t}}
\end{gathered}
$$

$u\left(R_{m}\right)$ : Combined Standard measurement uncertainty of recovery

$u\left(C_{o b s}\right):$ Standard measurement uncertainty of analysis of spiked sample

$u\left(C_{c e r t}\right)$ : Standard measurement uncertainty spiked sample

$R_{m}$ : Mean recovery

$C_{o b s}$ : Obtained concentration by analysis spiked sample

$C_{c e r t}$ : Spiked concentration

\subsubsection{Combined and expanded measurement uncertainty of the method}

Consequently, the combined standard measurement uncertainty of the method was calculated by Equation 8 . In order to obtain expanded uncertainty, combined standard measurement uncertainty has to be multiplied by 2 (coverage factor) at $95 \%$ confidence level. Relative measurement uncertainty values are given in Table 4.

$$
\frac{u_{c}(A)}{C_{A}}=\sqrt{\left(\frac{u\left(W_{S I}\right)}{W_{S I}}\right)^{2}+\left(\frac{u\left(W_{F S}\right)}{W_{F S}}\right)^{2}+\left(\frac{u\left(W_{I S S}\right)}{W_{I S S}}\right)^{2}+\left(\frac{u\left(C_{N S S}\right)}{C_{N S S}}\right)^{2}+\left(\frac{u\left(C_{0}\right)}{C_{0}}\right)+u\left(R_{m}\right)^{2}+u(r)^{2}}
$$

where,

$u_{c}(A)$ : Combined standard measurement uncertainty of analyte

$C_{A}$ : Concentration of analyte

$u\left(W_{S I}\right)$ : Combined standard measurement uncertainty of weighing sample intake

$W_{S I}$ : Weight of sample intake

$u\left(W_{F S}\right)$ : Combined standard measurement uncertainty of weighing final sample

$W_{F S}$ : Weight of final sample

$u\left(\mathrm{C}_{N S S}\right)$ : Combined standard measurement uncertainty of native stock solution

$C_{N S S}$ : Concentration of native stock solution

$u\left(c_{0}\right)$ : Combined standard measurement uncertainty of calibration curve 
$c_{0}$ : Determined concentration of the sample by using calibration curve

$u(r)$ : Standard measurement uncertainty of repeatability

$u\left(R_{m}\right)$ : Combined Standard measurement uncertainty of recovery

\section{Application of the method on hazelnut samples}

The samples of hazelnut shells and husks were collected at various altitudes from 19 sites in five different provinces and their methanol solution extracts were analyzed using LC-MS $\backslash$ MS technique. The amounts of paclitaxel, baccatin III, cephalomannine and 10-DAB III were determined from secondary plant metabolites of taxane class components. The results are presented in Table 5.

\section{Results and discussion}

\subsection{Method development}

This study presents a reliable LC-MS/MS method for the quantification of taxanes in hazelnut shells and husks. During method development, different conditions were examined to obtain high efficiency on extraction. Different type of solvents like ethanol, methanol, acetone and dichloromethane at different conditions were applied. Since the extraction performed with acetone at the ratio of 1:10 $(\mathrm{g} / \mathrm{mL})$ for $72 \mathrm{~h}$ in hazelnut shells was resulted with higher efficiency, it was concluded that, this extraction way is suitable for determination of paclitaxel in the hazelnut. The application of dichloromethane did not produce any result for hazelnut shells. In case of nut husks, only the usage of methanol at the ratio of $1: 15(\mathrm{gm} / \mathrm{L})$ for $72 \mathrm{~h}$ was efficient on determination of paclitaxel and cephalomannie. The biggest amount of baccatin III was obtained when the acetone has been applied at the ratio of 1:10 (gm/L) for $48 \mathrm{~h}$.

\subsection{Application of method on hazelnut sample}

Hazelnut shell and husk samples were collected at various altitudes from 19 sites in five different provinces, extracts of the samples were analyzed by LC-MS $\backslash$ MS technique.

The analyses indicated that while paclitaxel, cephalomannine and 10-DAB III were not present in nutshells, the highest amount of baccatin III, was found in the nutshell, collected at 250-500 $\mathrm{m}$ in the Cumayeri district of Düzce Province (Figure 6). The amount of Baccatin III in the nutshells was found to be more than in the husks samples (Table 5).

It can be concluded based on the Figure 4 and 5 that the concentration of analyte increases while the altitude value decreases. The highest amount of baccatin III was obtained in nutshells from regions, having the altitudes between $250-500 \mathrm{~m}$. Similar output was obtained in a study carried out in Italy. The regional variation in taxanes of Taxus species, the taxane in the European species of Taxus was analyzed, and the amounts of paclitaxel, 10-DAB III and taxane were detected in samples collected from 11 different locations. Although the paclitaxel was not detected, the highest amount of 10-DAB III was measured as $2277 \mathrm{mg} / \mathrm{kg}$ at $700 \mathrm{~m}$ altitude, whereas only $284 \mathrm{mg} / \mathrm{kg}$ taxane was measured at $1200 \mathrm{~m}$ altitude [11].

In case of husk samples, paclitaxel, cephalomannine and 10-DAB III were not detected but the high amount of baccatin III was obtained as well in those obtained in Vakfikebir district of Trabzon Province (Figure 5). Also the higher amount of baccatin III was detected in husk samples, region 13 at $0-250 \mathrm{~m}$ altitude as $923.64 \mu \mathrm{g} / \mathrm{kg}$ (Table 5). As a result, baccatin III was obtained in between 166.12$923.64 \mu \mathrm{g} / \mathrm{kg}$ in husk samples. Hoffmann et al. collected the tombul hazelnuts from Giresun, Turkey and made quantification of 10-DAB III, paclitaxel, cephalomannine and baccatin III in nutshell and husk. and they only reported the baccatin III in husk samples in between 1.10-67.7 $\mu \mathrm{g} / \mathrm{g}$ [18]. The difference between the mass fraction levels of baccatin III obtained in these two studies can originate from the difference of the region of hazelnuts.

Since the importance of baccatin III as precursor compound has been described in a number of studies [12, 18-21] detection and quantification of baccatin III in hazelnut was accomplished in this study. The hundred thousand tons of hazelnut were produced in every year in Turkey and so these products can be used as an alternative source of the paclitaxel precursor baccatin III. 
Determination of taxanes by validated LC-MS/MS method

\section{Conclusion}

This study presents a new and reliable LC-MS/MS method for the quantification of taxanes in hazelnut shells and husks. The extraction performed with acetone at the ratio of $1: 10\left(\mathrm{gmL}^{-1}\right)$ for $72 \mathrm{~h}$ in hazelnut shells was resulted with higher efficiency. After optimization, the reliability of the method was evaluated by method validation and uncertainty calculations. In validation process linearity, limit of detection (LOD), limit of quantification (LOQ) and repeatability (in a day and intra days) and recovery parameters were evaluated. The limit of detection values were obtained in between 0.94-2.14 $\mu \mathrm{g} / \mathrm{kg}$. The method has linear response in between $10-1000 \mu \mathrm{g} / \mathrm{kg}$ concentration levels. In evaluation of measurement uncertainty bottom-up approach was used. Each uncertainty sources were identified and evaluated. The relative measurement uncertainty values of the developed method were obtained between 2.1-7.6\%.

The method was applied on real sample which were collected in 19 different regions and altitudes. In nutshell and husk samples paclitaxel, cephalomannine and 10-DAB III were not detected. The highest amount of baccatin III, was found in the shells of nuts, collected at $250-500 \mathrm{~m}$ in the Cumayeri district of Düzce Province, it was also determined in husk samples collected at Vakfikebir district of Trabzon Province. The amount of taxane in the nutshells was determined to be more than in the husks samples.

Although the amounts of taxanes looks like in low level, these compounds acquired from natural products should be considered in light of the annual production efficiency exchange. The quantity of taxanes in hazelnut was determined in a very low level but the production of hazelnut shells and husks in hundred thousand tons each year can compensate that low concentration. In the industrial aspect, hazelnut shells and husks may be used as good and alternative sources of taxol if an efficient extraction process develops.

\section{Acknowledgment}

This study is supported by a grant (Project Number:114Z233) from Scientific and Technological Research Council of Turkey (TUBITAK)

\section{Conflict of Interest}

There is no conflict interest between authors.

\section{Supporting Information}

Supporting Information accompanies this paper on http://www.acgpubs.org/JCM

\section{ORCID}

Sibel Bay1l Oguzkan: 0000-0003-0254-6915

Bora Karagul: 0000-0002-0834-6183

Elif Sine Aksoy: 0000-0003-1177-0978

Ayşe Uzun: 0000-0001-9864-5744

Merve Can: 0000-0002-7005-1300

Hasibe Y1lmaz: 0000-0002-1308-1650

Halil Ibrahim Ugras: 0000-0002-1633-967X

Burcu Binici: 0000-0002-9616-8320

Ahmet C Gören:0000-0002-5470-130X 


\section{References}

[1] N. Erdemoğlu and B.Şener (1999). The biosynthesis of taxol and derivatives, J. Fac. Pharm., 28(2), 99-116.

[2] R.A. Holton, C. Somoza, H. Kim, F. Liang and J. Biediger (1994). First total synthesis of Taxol. 1. Functionalization of the B Ring, J. Am. Chem. Soc., 116, 1597-8.

[3] K.C. Nicolaou, Z. Yang, J.J. Liu, H. Ueno, P.G. Nantermet and R.K. Guy (1994). Total synthesis of Taxol, Nature, 367, 630-4.

[4] S.J. Danishefsky, J.J. Masters, W.B. Young, J.T. Link and L.B. Snyder (1996). Total synthesis of Baccatin III and Taxol, J. Am. Chem. Soc., 118, 2843-59.

[5] A. Paul and Wender (1997). The pinene path to taxanes. 5. Stereocontrolled synthesis of a versatile taxane precursor, J. Am. Chem. Soc., 119(11), 2755-2756.

[6] G.M. Cragg, S.A. Schepartz, M. Suffness and M.R.Grever (1993). The Taxol supply crisis. New NCI policies for handling the large-scale production of novel natural product anticancer and anti-HIV agents, $J$. Nat. Prod., 56(10), 1657-68.

[7] M. Colin, D. Guenard, F. Gueritte-Voegelein and P.Potier (1988). Preparation of Taxol derivatives as antitumor agents, Eur. Pat. Apll., EP 253738 Al.

[8] F. Gueritte-Voegelein, V. Senilh, B. David, D. Guenard, P. Potier (1986). Chemical Studies of $10-$ Deacetylbaccatin III. Hemisynthesis of Taxol Derivatives, Tetrahedron, 42, 4451-60.

[9] M.E. Wall and M. Wani (1998). History and future prospects of Camptothecin and Taxol in the Alkaloids, Academic Press, New York, 509-36.

[10] T. Mroczek and K. Glowniak (2001). Solid-phase extraction and simplified high performance liquid chromatography determination of 10-deacetylbaccatin III and related taxoid in yew species, J. Pharm. Biomed. Anal., 26, 89-102.

[11] M. Bollero, M.C. Loi, E.L.M. Rozendaal, T.A. Beek and C. Haar (2003). Analysis of pharmaceutically relevant taxoids in wild yew trees from Sardinia, Fitoterapia, 74, 34-39.

[12] R.G. Kelsey, N.C. Vance (1992). Taxol and cephalomannine concentrations in the foliage and bark of shade grown and sun-exposed Taxus brevifolia trees, J. of Nat. Prod., 55, 912-917.

[13] EURACHEM Guide, The Fitness for Purpose of Analytical Methods-A Laboratory Guide to Method Validation and Related Topics, Second Edition, (2014).

[14] H.O. Hamad, M. H. Alma, İ. Gulcin, M.A. Yılmaz and E. Karaoğul (2017). Evaluation of phenolic contents and bioactivity of root and nutgall extracts from Iraian Quercus infectoria Olivier, Rec. Nat. Prod. 11, 205210.

[15] A.C. Gören, G. Bilsel, M. Bilsel, K.S. Yenisoy and D. Karakaş (2004). Simple high-performance liquid chromatographic method for determination of atropine andobidoxime in a parenteral injection device, $J$. Chromatogr. A, 1057, 237-239.

[16] GUM, Evaluation of Measurement Data-Guide to the expression of uncertainty in measurement, (2008).

[17] EURACHEM/CITAC Guide CG4, Quantifying Uncertainty in Analytical Measurement, Second Edition, (2000).

[18] A. Hoffman and F. Shahidi (2009). Paclitaxel and other taxanes in hazelnut, J. Func. Foods I, 33-37.

[19] D.G.I. Kingston, A.G. Chaudhary, A.A.L. Gunatilaka and M.L. Middleton (1994). Synthesis of taxol from baccatin III via an oxazoline intermediate, Tetrahedron Lett. 35(26), 4483-4484.

[20] D.G.I. Kingston and E. Baloglu (1999). A new semisynthesis of Paclitaxel from Baccatin II, J. Nat. Prod., 62(7), 1068-1071.

[21] R. Holton (1990). Eur Pat. Appl. 400 971, US Patent no. 5015744 (1991); Eur. Pat. Appl. 428375 (1991); US Patent no. 5175315 (1992).

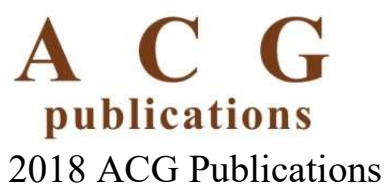

